\title{
PHYSIOLOGICAL CONCENTRATIONS OF UNCONJUGATED BILIRUBIN PREVENT OXIDATIVE STRESS-INDUCED HEPATOCANALICULAR DYSFUNCTION AND CHOLESTASIS
}

Cecilia L. Basiglio ${ }^{1,2}$, Flavia D. Toledo ${ }^{1}$, Andrea C. Boaglio ${ }^{1}$, Sandra M. Arriaga $^{2}$, Justina E. Ochoa ${ }^{1}$, Enrique J. Sánchez Pozzi ${ }^{1}$, Aldo D. Mottino ${ }^{1}$, Marcelo G. Roma ${ }^{1}$

${ }^{1}$ Instituto de Fisiología Experimental (IFISE-CONICET), ${ }^{2}$ Area Bioquímica ClínicaFacultad de Ciencias Bioquímicas y Farmacéuticas (U.N.R.), Rosario, Argentina

Author for correspondence:

Dr. Marcelo G. Roma

Instituto de Fisiología Experimental (IFISE)

Facultad de Ciencias Bioquímicas y Farmacéuticas

Suipacha 570, 2000 - Rosario, ARGENTINA

Tel.: +54-341-4305799

Fax: +54-341-4399473

E-mail: mroma@fbioyf.unr.edu.ar 


\section{ABSTRACT}

Bilirubin is an endogenous antioxidant with cytoprotective properties, and several studies highlight its potential in the treatment of pro-oxidant diseases. We demonstrated that oxidative stress (OS), a key feature in most hepatopathies, induces cholestasis by actin cytoskeleton disarrangement and further endocytic internalization of key canalicular transporters, such as the bile salt export pump (Bsep) and the multidrug resistance-associated protein 2 (Mrp2). Here, we evaluated the capability of physiological concentrations of unconjugated bilirubin (UB) to limit OS and the impairment in biliary secretory function induced by the model pro-oxidant agent, tertbutylhydroperoxide $(\mathrm{BuOOH})$. UB fully prevented the formation of reactive oxygen species (ROS) and membrane lipid peroxidation induced by $t \mathrm{BuOOH}$ in isolated rat hepatocytes. In the isolated rat hepatocyte couplet model, UB $(17.1 \mu \mathrm{M})$ prevented the endocytic internalization of Bsep and Mrp2 and the impairment in their secretory function induced by $t \mathrm{BuOOH}$. UB also prevented actin disarrangement, as evaluated by both plasma membrane bleb formation and actin fluorescent staining. Finally, UB prevented $\mathrm{tBuOOH}-$ induced $\mathrm{cPKC}$ activation. Experiments in isolated perfused rat livers showed that UB prevents the increase in oxidized glutathione biliary excretion and the drop in bile flow and the biliary excretion of specific Bsep and Mrp2 substrates. We conclude that physiological concentrations of UB are sufficient to prevent the biliary secretory failure induced by OS, by counteracting actin disarrangement and the consequent internalization of canalicular transporters relevant to normal bile formation. This reveals an important role for UB in preserving biliary secretory function under OS conditions. 
Basiglio et al

KEY WORDS: Unconjugated bilirubin, oxidative stress, hepatocellular cholestasis, canalicular transporters. 


\section{INTRODUCTION}

Due to its multiple energy-dependent functions, liver has a high mitochondrial metabolic rate, and is heavily engaged in detoxification mechanisms that involve redoxenzyme systems. Since these are major sources of endogenous free radicals, reactive oxygen species (ROS) production is higher in liver as compared with most organs. To counterbalance this oxidative challenge, hepatocytes are equipped with abundant antioxidant defenses that are critical to cellular integrity, including reduced glutathione and the redox enzymes catalase, glutathione peroxidase, and superoxide dismutase(Cessaratto et al. 2004). This borderline equilibrium makes liver highly susceptible to the pro-oxidant injury induced by pathological conditions that disrupt this balance by either or both increments in ROS formation and impairment of antioxidant defenses. Indeed, oxidative stress (OS) is a common feature in most hepatopathies, including, among others, hepatic ischemia-reperfusion injury following hepatectomy or liver transplantation, obstructive cholestasis, chronic cholestatic liver diseases, sepsisinduced cholestasis, viral, toxic, and autoimmune hepatitis, alcoholic and non-alcoholic steatohepatitis, and pathologies leading to hepatic accumulation of heavy metals, such as iron (hemochromatosis, iron-loading anemia) or copper (Wilson's disease) (Cessaratto et al. 2004, Copple et al. 2010).

In recent years, evidence has accumulated that OS is cholestatic in nature (Roma and Sanchez Pozzi 2008). Schmitt et al.(2000) and Kawai et al. (2007) observed a rapid decrease in bile flow, resulting in a complete cholestasis, after 60 min of perfusion of rat livers with $500 \mu \mathrm{M}$ of the pro-oxidant agent tert-butylhydroperoxide $(t \mathrm{BuOOH})$. By

using isolated rat hepatocyte couplets, an in vitro, polarized model to study hepatocanalicular secretory function, our group demonstrated that endocytic 
internalization of canalicular transporters is a key factor in this phenomenon. We have previously shown in isolated hepatocyte couplets that $t \mathrm{BuOOH}$ induces endocytic internalization of the bile salt export pump (Bsep), a main canalicular transporter that transports osmotically active monoanionic bile salts into the bile canaliculus, the main driving forces involved in the generation of the so called bile-salt independent bile flow (Pérez et al. 2006a). The same holds true for multidrug resistance-associated protein 2 (Mrp2), as has been shown in hepatocytes exposed to the pro-oxidant agents $t \mathrm{BuOOH}$ (Schmitt et al. 2000) and chloro-dinitro benzene (Schmitt et al. 2000); this transporter apically exports oxidized (GSSG) and reduced (GSH) glutathione (Yang et al. 2001), the main driving forces for the so called bile-salt-independent bile flow (Ballatori and Truong 1992). Previous studies from our laboratory in isolated rat hepatocyte couplets demonstrated that the mechanism underlying the internalization of canalicular transporters under OS conditions involves actin cytoskeleton disarrangement. Actin redistributes from pericanalicular zone to the rest of cell body, with the consequent formation of plasma membrane blebs (Pérez et al. 2006a). This phenomenon is triggered by a dysregulation of intracellular calcium levels, and the consequent activation of calcium-dependent protein kinase C (cPKC) (Pérez et al. 2006a,b). The extent to which these in vitro studies reproduce the actual situation in vivo is limited though, since cells are exposed to an extracellular medium lacking important cholephilic endogenous compounds, such as bile salts and bilirubin, which may have regulatory and even protective effects on the hepatocyte.

Bilirubin is the end product of heme degradation in mammals.By the concerted action of the enzymes heme oxygenase 1 and biliverdin reductase, heme molecule is degraded into iron, carbon monoxide, and biliverdin, which is subsequently converted 
into bilirubin (Maines 1997). Once formed, unconjugated bilirubin (UB) is carried in blood in the form of a reversible complex with serum albumin (McDonagh 2010). In liver, UB is converted into a series of water soluble derivatives by conjugation of one or two of its propionyl groups with glucuronic acid, glucose or xylose (Fevery 1977), before being excreted into bile, via Mrp2 (Nies 2007).

Although long considered a toxic waste product of heme catabolism, bilirubin has emerged as an important protective molecule in circulation. Previous in vitro studies demonstrated that bilirubin is an efficient antioxidant under physiological conditions (Stocker et al. 1987a, Sedlak et al. 2009). The antioxidant effects of bilirubin in vivo include scavenging of peroxyl radicals (Witting et al. 1996), inhibition of membrane lipid peroxidation (Witting et al. 1996), and scavenging of radical nitrogen species (Kaur et al. 2003). Albumin-bound bilirubin, in its unconjugated form, bears an extended system of conjugated double bonds and a pair of reactive hydrogen atoms which are most likely involved in bilirubin antioxidant action, by hydrogen donation to a radical species (Stocker 2004). Notably, binding of UB to albumin increases its radical scavenging action, in a reaction by which UB is stoichiometrically converted into its metabolic precursor, biliverdin (Stocker et al. 1987b). It is known that bilirubin greatly contributes to antioxidant defenses, due to its potent and versatile antioxidant activity. Moreover, several studies put forward the potential of UB in the treatment of cardiovascular (Ryter et al. 2007) and pulmonary (Ryter et al. 2007) diseases, as well as in the post-transplantation ischemia-reperfusion injury (Ollinger et al. 2007) and the immune response (Morita et al. 2003).These assumptions regarding the beneficial effects of bilirubin have been confirmed and extended by our previous studies on the 
Basiglio et al

hepatoprotective effects of UB on complement system-mediated cell lysis (Arriaga et al. 2009, Basiglio et al.2007 and 2010).

Bearing in mind the well-established antioxidant effects of UB, we hypothesize that UB may have a protective role against OS-induced cholestasis. Our results show that, even at physiological concentrations, UB has striking protective effects against OS-induced bile secretory failure, as assessed both in isolated rat hepatocyte couplets (IRHC) and in the isolated perfused rat liver (IPRL). 


\section{MATERIALS AND METHODS}

Materials.Collagenase type A (from Clostridiumhistolyticum), bovine serum albumin (BSA) fraction $\mathrm{V}, \mathrm{UB}$ (predominantly IX $\alpha$ isomer), $t \mathrm{BuOOH}$, thiobarbituric acid (TBA), dichlorofluorescein-diacetate (DCF-DA), taurocholate and chloro-dinitro benzene (CDNB) were purchased from Sigma Chemical Co. (St. Louis, MO, USA). 5chloromethyl-fluorescein-diacetate (CMF-DA) and Alexa Fluor 568 phalloidin were obtained from Molecular Probes (Eugene, OR, USA). Rabbit anti-rat Bsep was from Kamiya Biomedical Co. (Seattle, WA, USA) and mouse anti-human MRP2 (M2III-6) was from Alexis Biochemicals (San Diego, CA, USA). Cy2-conjugated donkey antirabbit IgG (31458) and Cy2-conjugated goat anti-mouse IgG (31430) were obtained from Thermo Fisher Scientific, Inc. (Waltham, MA, USA). Cholyl-glycylamidofluorescein (CGamF) was a generous gift from Prof. Alan Hofmann (University of California, San Diego, USA). All the other chemicals were of the highest purity commercially available.

Animals. Adult male Wistar rats weighing 300-350 g were used throughout. Animals were maintained on a standard diet and water ad libitum, and housed in a temperatureand humidity-controlled room under a constant 12-hour light, 12-hour dark cycle. All animals received humane care according to the criteria outlined in the "Guide for the Care and Use of Laboratory Animals", written by the National Academy of Sciences and published by the National Institutes of Health (NIH publication 25-28, revised 1996).

Evaluation of $\boldsymbol{t B u O O H}$-induced OS. Hepatocytes were isolated from livers by the collagenase perfusion technique, using the method of Berry and Friend (Berry and Friend 1969), with minor modifications. The resulting preparation yielded $\sim 400$ - 
$600 \times 10^{6}$ hepatocytes per liver with high viability ( $\left.>90 \%\right)$, as assessed by the Trypan blue exclusion test (Baur et al. 1975).

The magnitude of OS induced by $t \mathrm{BuOOH}$ was evaluated both by measuring the generation of the lipid peroxidation product MDA and by assessing fluorimetrically intracellular ROS production.

MDA was measured by reaction with thiobarbituric acid, as described previously (Borgognone et al. 2005), followed by fluorimetrical HPLC detection of the MDAthiobarbituric acid adduct formed, according to the HPLC method of Fukunaga et al. (Fukunaga et al. 1993). To identify the optimal UB concentration for further studying its antioxidant potential, an UB concentration range from 1.7 to $85 \mu \mathrm{M}$ was tested.

ROS formation in isolated rat hepatocytes was assessed by using $2^{\prime}, 7^{\prime}$ dichlorofluorescein (DCFH) as a fluorophore; its non-fluorescent, parent compound, $2^{\prime}, 7^{\prime}$-dichlorofluorescin diacetate (DCF-DA), readily crosses the hepatocyte membrane and is metabolized by nonspecific esterases and oxidized to DCFH by intracellular ROS (Cathcart et al. 1983). DCFH was detected by flow cytometric analysis. For this purpose, $2 \times 10^{6}$-hepatocyte suspensions were preloaded with $5 \mu \mathrm{M}$ DCF-DA for $30 \mathrm{~min}$, washed twice, and resuspended in the incubation medium containing UB (17.1 $\mu \mathrm{M}$, in the presence of $3 \% \mathrm{BSA}$ ) or its vehicle for $15 \mathrm{~min}$. We monitored the fluorescence base line for $2 \mathrm{~min}$, and then $t \mathrm{BuOOH}$ was added to the medium to reach a final concentration of $100 \mu \mathrm{M}$. After that, we continued the fluorimetric detection for another 15-min period.

\section{Studies in IRHC.}


Couplets isolation, culture and treatments. IRHC were obtained according to the twostep collagenase perfusion procedure followed by centrifugal elutriation as described by Wilton et al (1991). The resulting preparation, containing $71 \pm 3 \%$ of couplets of high viability $(>97 \%)$, was incubated in Leibovitz-15 medium at $37^{\circ} \mathrm{C}$ for $4.5 \mathrm{~h}$ to allow for attachment and repolarization. After that, IRHC were incubated with UB (17.1 $\mu \mathrm{M}, 15$ min) in the presence of $3 \% \mathrm{BSA}$, and then treated with $t \mathrm{BuOOH}(100 \mu \mathrm{M}, 15 \mathrm{~min})$.

Assessment of biliary secretory function. Bsep and Mrp2 transport functions were evaluated by using the fluorescent bile salt analog CGamF(Maglova et al. 1995)and glutathione methylfluorescein (GS-MF), respectively; GS-MF is a fluorescent Mrp2 substrate derived from CMF-DA which diffuses passively, and is intracellularly metabolized by esterases and glutathione $S$-transferases(Roelofsen et al. 1998). For this purpose, cells were exposed to $0.3 \mu \mathrm{M}$ CGamF (Maglova et al. 1995) or 2.5 $\mu \mathrm{M}$ CMFDA (Roma et al. 2000) for $15 \mathrm{~min}$; after that, images were captured using a digital camera (Q-color5; Olympus America, Center Valley, PA, USA) under a fluorescence microscope (Zeiss Axiovert 25; Carl Zeiss LLC, Thornwood, NY, USA), and the hepatocanalicular secretory function was assessed by determining the proportion of IRHC ( $>200$ per preparation) accumulating the fluorescent compounds in their canalicular vacuoles, as described (Roma et al. 2000).

Assessment of actin cytoskeleton integrity. Bleb formation is a preliminary marker of actin cytoskeleton disruption (Gores et al. 1990). IRHC were focused under visible light, and blebbing was expressed as the percentage of IRHC exhibiting at least one bleb in either of the cells, referred to the total number of IRHC analyzed (Stone et al. 1994). Assessment of Bsep, Mrp2 and F-actin localization.Intracellular localization of Bsep, Mrp2 and actin was evaluated by confocal laser microscopy. 
For Bsep and Mrp2 immunostaining, cells were then incubated with anti-Bsep rabbit antibody (1:100) or with anti-Mrp2 mouse antibody (1:100) for $2 \mathrm{~h}$, followed by a further incubation with Cy2-labeled anti-rabbit (or anti-mouse) IgG donkey (or goat) antibody (1:200), for $40 \mathrm{~min}$. In another set of experiments, fluorescent labeling with Alexa Fluor 568 phalloidinof fixed, permeabilized cells was employed to visualize cellular distribution of F-actin cytoskeleton.

Once stained, the specimens for Bsep, Mrp2 and F-actin analysis were examined by confocal microscopy (Eclipse C1 Plus confocal microscope system; Nikon instruments Inc., Melville, NY, USA) and the images thus obtained were analyzed to quantify the total intensity of fluorescence within the couplet and, specifically, in the canalicular region (for Bsep and Mrp2) or in the pericanalicular region (for F-actin) (Crocenzi et al. 2003).

\section{Studies in IPRL.}

Isolated rat liver perfusion. Rat livers were perfused in situ in a non-recirculating system with Ringer buffer equilibrated with $5 \% / 95 \%$ (v/v) $\mathrm{CO}_{2} / \mathrm{O}_{2}$. The perfusion flow was $40 \mathrm{ml} / \mathrm{min}$ throughout the experiment. IPRL were first perfused with $2.1 \mu \mathrm{M}$ $\mathrm{UB} / 3 \% \mathrm{BSA}$ for $15 \mathrm{~min}$, and then with $75 \mu \mathrm{M} t \mathrm{BuOOH}$ for $15 \mathrm{~min}$. During the perfusion process, bile samples were collected for gravimetric assessment of bile flow (assuming a bile density of $1.0 \mathrm{~g} / \mathrm{ml}$ )and for chemical determination of glutathione. In some experiments, taurocholate $(2 \mu \mathrm{M})$ and CDNB $(0.5 \mu \mathrm{M})$ were added to the perfusion buffer for further assessment of Bsep and Mrp2 function, respectively. The viability of the liver was monitored by the release of lactate dehydrogenase into the perfusate outflow; experiments exhibiting activities over $20 \mathrm{U} / \mathrm{L}$ were discarded. 
Assessment of oxidized glutathione:total glutathione relation in bile. Bile samples were subjected to assessment of both oxidized (GSSG) and total glutathione (GSHt) by the recycling method of Tietze (1969), as modified by Griffith (1980). The GSSG/GSHt relation in bile is a sensitive marker of hepatic OS (Lauterburg et al. 1984).

Assessment of biliary secretion of bile salts anddinitrophenyl-glutathione (GS-DNP). Total bile salts were determined by the $3 \alpha$-hydroxysteroid dehydrogenase procedure, as described by Talalay (1960). Biliary secretion of GS-DNP, a model Mrp2 substrate, was determined by HPLC.

Localization studiesof Bsep, Mrp2 and F-actin. Confocal microscopy was used to visualize both internalization of Bsep and Mrp2 from the canalicular domain and organization of actin cytoskeleton. Briefly, liver samples, obtained $20 \mathrm{~min}$ after the onset of $t \mathrm{BuOOH}$ perfusion, were prepared with a Zeiss Microm HM500 microtome cryostat (Carl Zeiss LLC, Thornwood, NY, USA). For Bsep and Mrp2 labeling, tissue sections were incubated overnight with the specific antibody to Bsep (1:100) or to Mrp2 (1:100). Sections were incubated either with Cy2-conjugated donkey anti-rabbit IgG $(1: 100)$ or with Cy2-conjugated goat anti-mouse $\operatorname{IgG}(1: 100)$ and with Alexa Fluor 568 phalloidin for F-actin staining for $1 \mathrm{~h}$.

Western blot analysis of PKCa activation. After culture for $24 \mathrm{~h}$, isolated rat hepatocytes were exposed to $t \mathrm{BuOOH}(100 \mu \mathrm{M})$ for $15 \mathrm{~min}$, washed with cold $0.3 \mathrm{M}$ sucrose, resuspended in $0.3 \mathrm{M}$ sucrose plus protease inhibitors $(25 \mu \mathrm{g} / \mathrm{mL}$ leupeptin, 5 $\mu \mathrm{g} / \mathrm{mL}$ pepstatin $\mathrm{A}$ and $0.1 \mathrm{mM}$ PMSF), and disrupted by sonication. In separate experiments, we tested the effect of UB $(17.1 \mu \mathrm{M})$ by exposing the cells for $15 \mathrm{~min}$ to the pigment, previous to the incubation with $t \mathrm{BuOOH}(100 \mu \mathrm{M})$ for $15 \mathrm{~min}$ or its solvent. Cytosolic (supernatant) and total membrane (pellet)-enriched fractions were 
obtained by ultracentrifugation for $60 \mathrm{~min}$ at $100,000 \times \mathrm{g}$ (Carreras et al. 2003) and total protein determined (Lowry et al. 1951). Proteins were separated by electrophoresis on a 12\%-SDS-polyacrylamide gel and after electrotransfer, nitrocellulose membranes (Immobilon-P membranes) were incubated overnight with a mouse monoclonal antibody to PKCa (BD Biosciences Pharmingen; 1:1,000). Stripped membranes were reprobed with a mouse anti- $\beta$ actin antibody (Sigma, 1:5,000, $1 \mathrm{~h}$ ). After incubation with a donkey anti-mouse IgG secondary antibody (Thermo Fisher, 1:5000, 1h), a chemiluminescence reagent, and Hyperfilm ECL, PKC $\alpha$ and $\beta$ actin bands were quantified by densitometry with ImageJ $1.44 \mathrm{~m}$ (National Institutes of Health) software. To estimate the amount of PKC $\alpha$ associated with both cytosolic and membrane fractions, the relative intensity of each PKC $\alpha$ band was related to the correspondent $\beta$ actin band, divided by the $\mu \mathrm{g}$ of protein loaded in that lane, and then multiplied by the total amount of protein recovered in the corresponding fraction. The proportion of membrane-bound PKC $\alpha$ was expressed as the amount in membranes relative to the total (membrane-bound + cytosolic) amount the protein.

\section{Statistical analysis.}

Results were expressed as mean \pm S.E.M. When requirements for parametric analysis were met, a Student's unpaired $t$ test was used for comparison between two groups; comparisons that did not meet this criterion were carried out using the MannWhitney's rank sum test. The Kruskal-Wallis' test (one-way ANOVA by ranks) was used when more than two groups were compared, followed by the Dunn's multiplecomparison, post hoc test for pairwise comparisons, if ANOVA reached any statistical significance among groups. $p$ values lower than 0.05 were judged to be significant. The 
Basiglio et al

variances of the densitometric profiles of Bsep and Mrp2 localization were compared with the Mann-Whitney $U$ test. $p$ values $<0.05$ were considered to be statistically significant. 


\section{RESULTS}

Effect of $U B$ on the oxidative damage induced by $\mathrm{tBuOOH}$ in isolated rat hepatocytes. Hepatocytes were incubated with or without UB (1.7-85 $\mu \mathrm{M}, 15 \mathrm{~min})$ and then with $t \mathrm{BuOOH}(100 \mu \mathrm{M}, 15 \mathrm{~min})$. We found that concentrations of UB up to $42.5 \mu \mathrm{M}$ fully prevented formation of malondialdehyde (MDA), while higher concentrations did not (Figure 1, panel $a$ ). This finding prompted us to continue studying the antioxidant capacity of UB using the physiological concentration of $17.1 \mu \mathrm{M}$. We found that ROS formation was completely prevented by UB (Figure 1, panel $b$ ). These results indicate acytoprotective action of UB on the pro-oxidant effects of $t \mathrm{BuOOH}$. UB had no direct effect on basal oxidative status, since hepatocytes incubated only with UB showed similar levels of ROS and MDA content to control cells.

\section{Effect of $U B$ on the impairment of function and localization of the canalicular} transporters Bsep and Mrp2 induced by tBuOOH in IRHC. After incubation with UB $(17.1 \mu \mathrm{M}, 15 \mathrm{~min})$ and $t \mathrm{BuOOH}(100 \mu \mathrm{M}, 15 \mathrm{~min})$, we evaluated the percentage of IRHC able to accumulate the fluorescent substrates CGamF or GS-MF in their canalicular vacuole. Control IRHC exposed only to BSA (UB vehicle) and DMSO $(t \mathrm{BuOOH}$ vehicle) showed a 55-60\% accumulation of either fluorescent substrates. We found that, while $t \mathrm{BuOOH}$ caused a decrease in the percentage of couplets accumulating the fluorescent substrates in their vacuoles, UB fully prevented thisdecrease (Figure 2, panel $a)$.

To elucidate whether the functional impairment of canalicular transporters induced by $t \mathrm{BuOOH}$ was due to a relocalization of such transporters from the 
canalicular membrane to submembraneous vesicles, we carried out localization studies by confocal microscopy followed by image analysis. In control IRHC, both Bsep and Mrp2 were mainly confined to the canalicular membrane, while $t \mathrm{BuOOH}(100 \mu \mathrm{M})$ induced relocalization of both transporters into vesicles localized all over the couplet body. Redistribution of Bsep and Mrp2 was fully andpartially prevented by UB (17.1 $\mu \mathrm{M})$, respectively. UB did not have any independent effect on localization of these transporters (Figure 2, panel $b$ ).

Effect of $U B$ on bleb formation and F-actin redistribution induced by tBuOOH. To assess whether endocytic internalization of Bsep and Mrp2 was due to actin cytoskeleton disarrangement, bleb formation was assessed as a preliminary marker of actin cytoskeleton disruption (Gores et al. 1990). $t \mathrm{BuOOH}(100 \mu \mathrm{M})$ induced extensive plasma membrane blebbing (as visualized by phase-contrast microscopy), while UB $(17.1 \mu \mathrm{M})$ almost fully prevented this event (Figure 3, panel $a$ ). F-actin distribution under the same conditions used to study Bsep and Mrp2 localization was also assessed (Figure 3, panel $b$ ). In control couplets, F-actin displayed pericanalicular localization, forming a dense belt around the canalicular membrane. $t \mathrm{BuOOH}$ induced extensive redistribution of F-actin from the pericanalicular area to both the cell body and the sinusoidal plasma membrane, particularly in the areas where blebs occurred. As with transporters localization and bleb formation, redistribution of F-actin was almost fully prevented by pretreatment of IRHC with UB. These findings strongly support the notion that UB prevents oxidative biliary secretory failure by preventing the canalicular transporters endocytosis that occurs as a consequence of actin cytoskeleton disruption. 
Effect of $U B$ on the oxidative damage induced by $t B u O O H$ in IPRL. After isolation, livers were perfused first with UB $(2.1 \mu \mathrm{M}, 15 \mathrm{~min})$ or its vehicle $(3 \% \mathrm{BSA})$, and then with $t \mathrm{BuOOH}(75 \mu \mathrm{M}, 15 \mathrm{~min})$. Ancillary experiments aimed at setting the conditions for liver perfusion showed that $2.1 \mu \mathrm{M}$ and $75 \mu \mathrm{M}$ were the optimal concentrations of $\mathrm{UB}$ and $t \mathrm{BuOOH}$ in this model, respectively. Oxidative injury was evaluated by measuring the GSSG/GSHt relation in bile. Time variations in this parameter were expressed as percentages of the value obtained at $t=0$ (onset of the perfusion process). While $t \mathrm{BuOOH}$ induced a sharp and transient increase in GSSG/GSHt relation, UB markedly attenuated this phenomenon (Figure 4, panel $a$ ).

Effect of $U B$ on bile flow impairment induced by $\mathbf{t B u O O H . ~ B i l e ~ f l o w ~ s h a r p l y ~ d r o p p e d ~}$ after perfusion with $t \mathrm{BuOOH}$, reaching a minimum at $20 \mathrm{~min}$ after the onset of the perfusion with the pro-oxidant agent. Pre-perfusion of IPRL with UB completely prevented the decrease in bile flow, so that its time course profile was similar to that of Control livers (Figure 4, panel $b$ ). UB per se did not cause any alterations in bile flow.

Effect of $U B$ on biliary secretion of Bsep and Mrp2 substrates. Since we found that bile flow was impaired by $t \mathrm{BuOOH}$ perfusion, we assessed whether Bsep and Mrp2 function was also impaired. For this purpose, in another set of experiments, taurocholate and CDNB were added to the perfusion solution at the beginning of the perfusion process. Time variations in the excretion of either substrates were expressed as percentages of the values obtained at $t=0$ (onset of the perfusion process). We found that $t \mathrm{BuOOH}(75 \mu \mathrm{M})$ induced a $70 \%$ decrease in the biliary excretion of taurocholate, a Bsep substrate, and that UB $(2.1 \mu \mathrm{M})$ partially prevented this decrease (Figure 4, panel 
c). Similarly, UB fully prevented the abrupt decrease in the biliary excretion of the Mrp2 substrate GS-DNP (Figure 4, panel $d$ ). Our results show that UB prevents the OSinduced functional impairment of both Bsep and Mrp2, the two key canalicular transporters involved in bile secretion.

Effect of UB on Bsep, Mrp2 and F-actin localization. Tissue sections obtained from IPRL experiments were subjected to specific co-staining of Bsep (green) and actin (red), or of Mrp2 (green) and actin (red). $t \mathrm{BuOOH}$ induced redistribution of both transporters from the canalicular membrane into vesicles located either in cytosol or around the pericanalicular area, as evidenced by an increase in colocalization of these transporters with pericanalicular actin (yellowish area). Aconcomitant disruption of actin cytoskeleton became also apparent, as shown by the distortion and discontinuity of the actin pericanalicular belt in the $t \mathrm{BuOOH}$ group, as compared with the control one. Preperfusion with UB almost fully prevented all these alterations(Figure 5). Taken together, these results strongly suggest that the protective action of UB on the oxidative impairment of biliary secretory function is exerted at the level of actin cytoskeleton integrity, and its consequent role in the proper canalicular localization of transporters that are crucial to the biliary secretion process.

Effect of $\boldsymbol{U B}$ on PKCa activation. Activation of $\mathrm{PKC} \alpha$, the main $\mathrm{cPKC}$ in hepatocytes, is associated with translocation of the protein from the cytosol to cellular membranes. Representative immunoblots of PKC $\alpha$ in both cytosolic and membrane fractions of primary cultured rat hepatocytes incubated with $100 \mu \mathrm{M} t \mathrm{BuOOH}$, in the presence or absence of $17.1 \mu \mathrm{M} \mathrm{UB}$, are shown in the upper panel of Figure 6. $t \mathrm{BuOOH}$ increased 
Basiglio et al

membrane-bound (active) PKC $\alpha$ by $50 \%$. This was fully prevented by pre-incubation of the cells with $17.1 \mu \mathrm{M}$ UB (lower panel of Figure 6). 


\section{DISCUSSION}

Bilirubin, historically considered a waste product of heme catabolic pathway and even a potentially toxic compound, is nowadays regarded as a potent antioxidant agent (Sedlak et al. 2009, Stocker et al. 1987a, Vitek 2012). This study provides new insights into the hepatoprotective properties of UB by showing that the pigment, when present at physiological concentrations, can preserve the functional status of hepatocytes in terms of biliary secretory function under OS conditions, serving as a crucial antioxidant defense to keep the secretory machinery in operation after an oxidative challenge.

We first tested the antioxidant effect of UB at physiological concentrations in a hepatocellular, in vitro model of oxidative-stress damage, as that induced by the model pro-oxidant agent $t \mathrm{BuOOH}$ to isolated hepatocytes; $t \mathrm{BuOOH}$ freely diffuses across the plasma membrane, and is converted into oxygen (peroxyl and alkoxyl) free radicals mainly by microsomal cytochrome P450 (Davies 1989) and, to a lesser extent, through reduction in the mitochondrial electron transport chain (O'Donnel and Burkitt 1994). We found that $t \mathrm{BuOOH}$ increased ROS formation in this model and that physiological concentrations of UB completely prevented this effect.

A possible mechanism underlying UB antioxidant action likelyinvolves the cycle between bilirubin and biliverdin catalyzed by the enzyme biliverdin reductase, which would allow a single bilirubin molecule to function as an antioxidant many times over(Sedlak and Snyder 2004, Liu et al. 2006). Indeed, the tissue concentrations of UB $(\sim 20-50 \mathrm{nM})$ are by far too low to cope with the mM levels of ROS that cells encounter physiologically, but due to the biliverdin-UB cycle, as little as $10 \mathrm{nM}$ UB protects against 10,000 higher fold concentrations of hydrogen peroxide(Doré et al. 1999).However, other authors have found that bilirubin oxidation yields little, if any, 
biliverdin (McDonagh 2010, Maghzal et al. 2009), thus favoring the hypothesis that bilirubin may simply act as a scavenger of ROS by blocking the initiation of a radical chain process (Stocker 2004). This may be particularly so in our cellular/tissular systems.In IRHC and IPRL experiments, UB was added to the incubation/perfusion solution in a 1:1 molar ratio with human serum albumin, but once inside the hepatocytes, UB bounds to the cytosolic organic anion-binding protein, glutathione transferase. Maghzal et al. demonstrated that, in a model of extracellular (albuminbound) bilirubin, oxidative conversion to biliverdin after an oxidative challenge is very efficient, but oxidation of glutathione transferase-bound bilirubin yields only modest amounts of biliverdin (2009).

The link between the well-recognized capability of UB to scavenge ROS in noncellular systems should not be extrapolated mechanically to isolated hepatocytes, or to the situation in vivo. Indeed, in a cellular-preserved context, UB has dual, antioxidant and pro-oxidant effects depending on its exposure levels. This is in line with our results showing that only physiological (i.e., up to $17.1 \mu \mathrm{M}$ ) UB concentrations significantly prevented $t \mathrm{BuOOH}$-induced lipid peroxidation, without further improvements at twice higher concentrations of the pigment, and loss of the protective effect at four-time higher concentrations (see Figure $1 a$ ). Protective effects of physiological UB levels against OS-induced hepatocellular damage are consistent with those recently published by Zelenka et al.(2012), who found that intracellular accumulation of UB derived from sodium arsenite-induced increase in heme production and further $\mathrm{HO}-1$ induction is associated with a dramatic decrease in lipid peroxidation in HepG2 cells. On the other hand, potential deleterious effects of higher levels of bilirubin is illustrated by the finding that up-regulation of HO-1 in rats subjected to either bile duct ligation (Froh 
etal. 2007) or $\mathrm{Cl}_{4} \mathrm{C}$ exposure (Eipel et al. 2007), two maneuvers inducing ROS-mediated hepatocellular damage, increase rather than attenuate liver injury, an effect that was prevented by HO-1 inhibitors.

The finding that $\mathrm{UB}$, at low levels, i.e. with such a high efficiency, is instrumental in preventing OS in hepatocytes, led us to speculate that physiological concentrations of bilirubin may protect hepatocanalicular secretory function from an oxidative challenge. If so, the finding may have implications to explain the paradoxical findings often described in the literature that, despite most liver diseases involve either or both high ROS production and impairment of antioxidant defenses, they are often non-cholestatic in nature. For example, viral hepatitis is characterized by OS generation due to a combination of chronic inflammation, iron overload, mitochondrial damage, and proteins encoded by the virus (Stehbens 2004, Choi and Ou 2006). However, few cases occur with overt cholestasis (e.g. 2\% in viral hepatitis A) (Petrov et al. 2012), and the incidence is even lower in viral hepatitis B and C (Davies et al. 1991). Another example is non-alcoholic fatty liver disease. Even when OS is critically involved in both onset and progression of the disease (Rolo et al. 2012), histological images reveal that the few patients displaying cholestatic features have ductular rather than hepatocellular damage (Sorrentino et al. 2005). The discrepancy between the relatively low cholestatic impact of OS in clinical hepatopathies and the well-established evidence that OS is highly cholestatic when studied in in vitro models (e.g. IRHC, HepG2 cell line, IPRL) may be due to the absence of extracellular UB in the latter experimental settings. Therefore, UB may be crucial to preserve ongoing bile secretion despite extensive occurrence of pro-oxidant mechanisms in liver disease. 
A key event leading to hepatocanalicular dysfunction under OS conditions is the disarrangement of the F-actin cytoskeleton. This structure is mainly localized in hepatocytes around the canalicular membrane, and critically influences transporter stability in this membrane domain. Actin can interact with, and possibly regulate, transmembrane proteins via binding to radixin, which acts as a cross-linker between canalicular transporters and actin filaments (Crocenzi et al. 2012). In line with this, the actin-cytoskeleton disrupting agent phalloidin triggers rapid internalization of Mrp2 (Rost et al. 1999), and mice lacking radixin develop conjugated hyperbilirubinemia associated with Mrp2 retrieval(Kikuchi et al. 2002). We have previously shown that the OS-mediated cytosolic $\mathrm{Ca}^{2+}$ increase and the further activation of $\mathrm{cPKC}$ are involved in F-actin disarrangement, rather than direct oxidation of actin sulfhydryl groups (Pérez et al. 2006a,b). Here, we demonstrated that UB helps to preserve F-actin integrity by hampering OS-mediated cPKC activation, because of its scavenging properties.

UB is highly lipophilic, and therefore it preferentially protects cell membranes, including the mitochondrial ones, from lipid peroxidation (Sedlak et al. 2009); UB is even more efficient than $\alpha$-tocopherol, which is regarded as the best exogenous antioxidant against lipid peroxidation (Stocker et al. 1987a, Mancuso et al. 2012). Low OS levels, like those developed under our experimental conditions, selectively impair mitochondrial function. At the concentrations used here, $t \mathrm{BuOOH}$ was shown to cause mitochondrial pyridine nucleotide oxidation, which disrupts the balance of mitochondrial $\mathrm{Ca}^{2+}$ uptake and release, leading to a net increase of mitochondrial $\mathrm{Ca}^{2+}$; the combination of increased $\mathrm{Ca}^{2+}$ and ROS levels causes opening of the mitochondrial permeability transition pores, which induces further OS and mitochondrial $\mathrm{Ca}^{2+}$ uptake (Byrne et al. 1999). Eventually, this opens ATP-sensitive, non-specific cation channels, 
thus leading to $\mathrm{Ca}^{2+}$ influx from the extracellular medium (Sato et al. 2009). Additionally, $t \mathrm{BuOOH}$-derived ROSmay induce direct activation of the reverse mode of the plasma membrane carrier, $\mathrm{Na}^{+} / \mathrm{Ca}^{2+}$ exchanger(Kim et al. 2000). It is therefore tempting to speculate that bilirubin is the "first line of defense" to hamper these membrane-based processes, by preventing both mitochondria dysfunction and oxidation of plasma membrane-integrated carriers, thus preventing the cytosolic $\mathrm{Ca}^{2+}$ rise and the further cPKC activation. Actually, we showed here that UB is indeed able to prevent activation of this harmful, pro-cholestatic signaling pathway.

Even when bilirubin can efficiently counteract the cascade of events involved in OS-induced hepatocanalicular dysfunction, actual protection against cholestasis induced by OS needs to be confirmed, as we did here. Indeed, the anticholestatic effects of UB may be limited by direct, deleterious effects of the pigment on the functional status of canalicular transporters. Large loads of UB to experimental animals induce cholestasis, due to a marked impairment of Bsep activity by direct perturbations on the plasma membrane environment (Labori et al. 2002). Our finding that UB restored to normality not only the localization status but the functional activity of both Bsep and Mrp2, as shown both in the IRHC and the IPRL models using specific substrates (see Figures 2 and 4, respectively), indicates that the levels of UB required to afford protection against a cholestatic oxidant challenge are not harmful but protective to biliary secretory function.

There is developing evidence in the literature that UB can counteract oxidativestress mediated cholestasis, although their putative beneficial effects are related to late improvements in canalicular transporters expression rather than prevention of acute changes in localization, as demonstrated here. The evidence is also indirect, since it 
relays on manipulation of UB hepatocellular levels by modulation of HO-1 activity. For example, pre-induction of HO-1 with a hemoglobin analogue ameliorated cholestasis and downregulation of Bsep and Mrp2 in cholestasis induced by cold ischemiareperfusion of rat liver, an OS-related injury (Granato et al. 2003); however, other products derived from the $\mathrm{HO}-1$-catalyzed reaction, e.g. $\mathrm{CO}$, may have accounted for this protection. Our results showing conclusively that direct exposure to UB counteracts the cholestatic effects of OS help to support the contention that changes in HO-1 activity under cholestaticconditions, considered rather as an epiphenomenon in the context of the disease, may be a key protective mechanism as a source of protective, anticholestatic UB. Interestingly, bile salts, which are retained in cholestasis due to the biliary secretory failure, have been shown to upregulate HO-1(Donner et al. 2012), and cholestasis is associated with an enlargement of the free cytosolic pool of the HO-1 substrate heme (González et al.2011). It is therefore tempting to speculate that bile salts favor the production of an antioxidant (UB) against its own pro-oxidant effects when retained during a cholestatic process; this may attenuate the progression of the disease by breaking the vicious circle in which bile salts retained by the cholestatic process induce OS, which in turn induces more cholestasis. Furthermore, since expression of HO-1 is rapidly upregulated in the liver following any oxidative challenge, HO-1 induction may represent an adaptive response to mitigate the severity of the oxidative damage (Schacter et al. 1983), including the cholestatic one.

In conclusion, the data presented here demonstrate that physiological concentrations of UB prevent oxidative-stress induced hepatocellular damage. This cytoprotective effect is exerted through prevention of actin cytoskeleton disarrangement and the consequent endocytic internalization of canalicular transporters crucial to biliary 
secretory function. These findings support the notion that an endogenously generated metabolite, UB, may act as an efficient antioxidant agent at physiological (micromolar) concentrations. Whatever the molecular mechanism of UB antioxidant action is, it is clear from the data presented here that the pigment can neutralize ROS that are continuously being generated as a consequence of hepatocellular metabolic activity, and that this antioxidant effect may become highly relevant to preserve normal biliary function. Furthermore, since ROS become often increased in liver under a myriad of pathological conditions, and since UB often accumulates in tissues and plasma in these hepatopathies, we hypothesize that those hepatic diseases bearing an oxidative background would have a worse outcome in terms of hepatobiliary function in the absence of UB. This encourages further characterization of the therapeutic potential of UB manipulation in liver disease. 
Basiglio et al

\section{ACKNOWLEDGEMENTS}

This work was supported by grants from Agencia Nacional de Promoción Científica y Tecnológica (ANPCyT) and Consejo Nacional de Investigaciones Científicas y Técnicas (CONICET) to Drs. Aldo D. Mottino and Marcelo G. Roma. We thank Drs. Marcelo Luquita, Mara Ojeda and Rodrigo Vena for their valuable technical assistance in HPLC determinations, flow cytometric studies and confocal imaging, respectively.

\section{Conflict of interest statement}

The authors declare that they have no conflict of interest. 


\section{REFERENCES}

Arriaga SM, Basiglio CL, Mottino AD, Almará AM(2009) Unconjugated bilirubin inhibits C1 esterase activity. Clin Biochem 42:919-921

Ballatori N, Truong AT (1992) Glutathione as a primary osmotic driving force in hepatic bile formation. Am J Physiol (Gastrointest Liver Physiol) 263:G617-G624

Basiglio CL, Arriaga SM, Pelusa F, Almará AM, Roma MG, Mottino AD(2007) Protective role of unconjugated bilirubin on complement-mediated hepatocytolysis. Biochim Biophys Acta 1770:1003-1010

Basiglio CL, Arriaga SM, Pelusa F, Almará AM, Kapitulnik J, Mottino AD(2010) Complement activation and disease: protective effects of hyperbilirubinemia. Clin Sci 118:99-113

Baur H, Kasperek S, Pfaff E(1975) Criteria of viability of isolated liver cells. Hoppe Seylers Z Physiol Chem. 356:827-838

Berry N and Friend DS (1969) High-yield preparation of isolated rat liver parenchymal cells: a biochemical and fine structural study. J Cell Biol 43:506-520

Borgognone M, Pérez LM, Basiglio CL, Ochoa JE, Roma MG(2005) Signaling modulation of bile salt-induced necrosis in isolated rat hepatocytes. Toxicol Sci 83:114125 
Byrne AM, Lemasters JJ, Nieminen AL(1999) Contribution of increased mitochondrial free $\mathrm{Ca}^{2+}$ to the mitochondrial permeability transition induced by tertbutylhydroperoxide in rat hepatocytes. Hepatology 29:1523-1531

Carreras FI, Gradilone SA, Mazzone A, Garcia F, Huang BQ, Ochoa JE, Tietz PS, Larusso NF, Calamita G, Marinelli RA(2003) Rat hepatocyte aquaporin-8 water channels are down-regulated in extrahepatic cholestasis. Hepatology 37:1026-1033

Cathcart R, Schwiers E, Ames B. Detection of picolmole levels of hydroperoxides using a fluorescent dichlorofluorescein assay (1983) Anal Biochem 134:111-116

Cesaratto L, Vascotto C, Calliga,is S, Tell G(2004) The importance of redox state in liver damage. Ann Hepatol 3:86-92

Choi J, Ou JH. Mechanisms of liver injury.III(2006) Oxidative stress in the pathogenesis of hepatitis C virus. Am J Physiol (Gastrointest Liver Physiol) 290:G847G851

Copple BL, Jaeschke H, Klaassen CD (2010) Oxidative stress and the pathogenesis of cholestasis. Semin Liver Dis 30:195-204

Crocenzi FA, Mottino AD, Cao J, Veggi LM, Sanchez Pozzi EJ, Vore M, Coleman R, Roma M(2003) Estradiol-17-bD-glucuronide induces endocytic internalization of Bsep 
in rats. Am J Physiol Gastrointest Liver Physiol 285:G449-G459

Crocenzi FA, Zucchetti AE, Boaglio AC, Barosso IR, Sanchez Pozzi EJ, Mottino AD, Roma MG (2012) Localization status of hepatocellular transporters in cholestasis. Front Biosci 17:1201-1218

Davies MJ(1989) Detection of peroxyl and alkoxyl radicals produced by reaction of hydroperoxides with rat liver microsomal fractions. Biochem J 257:603-606

Davies SE, Portmann BC, O'Grady JG, Aldis PM, Chaggar K, Alexander GJ, Williams $\mathrm{R}(1991)$ Hepatic histological findings after transplantation for chronic hepatitis B virus infection, including a unique pattern of fibrosing cholestatic hepatitis. Hepatology $13: 150-157$

Donner M, Topp SA, Cebula P, Krienen A, Gehrmann T, Sommerfeld A, Reinehr R, Macher A, Herebian D, Mayatepek E, Pannen BH, Knoefel WT, Haeussinger D(2012) HbG200-mediated preinduction of heme oxygenase-1 improves bile flow and ameliorates pericentral downregulation of Bsep and Mrp2 following experimental liver ischemia and reperfusion. Biol Chem 394:97-112

Doré S, Takahashi M, Ferris CD, Zakhary R, Hester LD, Guastella D, Snyder SH(1999) Bilirubin, formed by activation of heme oxygenase-2, protects neurons against oxidative stress injury. Proc Natl Acad Sci USA 96:2445-2450 
Eipel C, Eisold M, Schuett H, Vollmar B(2007) Inhibition of heme oxygenase-1 protects against tissue injury in carbon tetrachloride exposed livers. J Surg Res 139:113120

Fevery J, Van de Vijver M, Michiels R, Heirwegh KP(1977) Comparison in different species of biliary bilirubin-IX alpha conjugates with the activities of hepatic and renal bilirubin-IX alpha-uridine diphosphate glycosyltransferases. Biochem J 164:737-746

Froh M, Conzelmann L, Walbrun P, Netter S, Wiest R, Wheeler MD, Lehnert M, Uesugi T, Scholmerich J, Thurman RG (2007) Heme oxygenase-1 overexpression increases liver injury after bile duct ligation in rats. World J Gastroenterol 13:34783486

Fukunaga K, Suzuki T, Takama K(1993) Highly sensitive high-performance liquid chromatography for the measurement of malondialdehyde in biological samples. $\mathrm{J}$ Chromatogr 621:77-81

González R, Cruz A, Ferrín G, López-Cillero P, Fernández-Rodríguez R, Briceño J, Gómez MA, Rufián S, Mata M de L, Martínez-Ruiz A, Marin JJ, Muntané J (2011) Nitric oxide mimics transcriptional and post-translational regulation during $\alpha$ tocopherol cytoprotection against glycochenodeoxycholate-induced cell death in hepatocytes. J Hepatol 55:133-144

Gores G, Herman B, Lemasters J(1990) Plasma membrane bleb formation and rupture: 
a common feature of hepatocellular injury. Hepatology 11:690-698

Granato A, Gores G, Vilei MT, Tolando R, Ferraresso C, Muraca M(2003) Bilirubin inhibits bile acid induced apoptosis in rat hepatocytes. Gut 52:1774-1778

Griffith OW(1980) Determination of glutathione and glutathione disulfide using glutathione reductase and 2-vinylpyridine. Anal Biochem 106:207-212

Kaur H, Hughes M, Green CJ, Naughton P, Foresti R, Motterlini R(2003) Interaction of bilirubin and biliverdin with reactive nitrogen species. FEBS Lett 543:113-119

Kawai S, Arai T, Yokoyama Y, Nagino M and Nimura Y(2007) Free oxygen radicals reduce bile flow in rats via an intracellular cyclic AMP-dependent mechanism.J Gastroenterol Hepatol 22:429-435

Kikuchi S, Hata M, Yamane Y, Matsui T, Tamura A, Yonemura S, Yamagishi H, Keppler D, Tsukita S and Tsukita S(2002) Radixin deficiency causes conjugated hyperbilirubinemia with loss of Mrp2 from bile canalicular membranes. Nat Genet $31: 320-325$

Kim JA, Kang YS, Lee SH, Lee YS (2000) Inhibitors of $\mathrm{Na}^{+} / \mathrm{Ca}^{2+}$ exchanger prevent oxidant-induced intracellular $\mathrm{Ca}^{2+}$ increase and apoptosis in a human hepatoma cell line. Free Radic Res 33:267-277 
Maines MD(1997) The heme oxygenase system: A regulator of second messenger gases. Annu Rev Pharmacol Toxicol37:517-554

Labori KJ, Arnkvaern K, Bjørnbeth BA, Press CM, Raeder MG(2002) Cholestatic effect of large bilirubin loads and cholestasis protection conferred by cholic acid coinfusion: a molecular and ultrastructural study. Scand J Gastroenterol 37:585-596

Lauterburg BH, Smith CV, Hughes H and Mitchell JR(1984) Biliary excretion of glutathione and glutathione disulfide in the rat. Regulation and response to oxidative stress. J Clin Invest 73:124-133

Liu Y, Liu J, Tetzlaff W, Paty DW, Cynader MS(2006) Biliverdin reductase, a major physiologic cytoprotectant, suppresses experimental autoimmune encephalomyelitis. Free Radic Biol Med 40:960-967

Lowry OH, Rosebrough NJ, Farr LL, Randall RJ(1951) Protein measurement with the Folin phenol reagent. J Biol Chem 193:265-275

Maghzal GJ, Leck M, Collinson E, Li C and Stocker R(2009) Limited Role for the Bilirubin-Biliverdin Redox Amplification Cycle in the Cellular Antioxidant Protection by Biliverdin Reductase.J Biol Chem 284:29251-29259

Maglova LM, Jackson AM, Meng XJ, Carruth MW, Schteingart CD, Ton-Nu HT, Hofmann AF, Weinman SA (1995) Transport characteristics of three fluorescent 
conjugated bile acid analogs in isolated rat hepatocytes and couplets. Hepatology $22: 637-647$

Mancuso C, Barone E, Guido P, Micelia F, Di Domenico F, Perluigi M, Santangelo R, Preziosi $\mathrm{P}(2012)$ Inhibition of lipid peroxidation and protein oxidation by endogenous and exogenous antioxidants in rat brain microsomes in vitro. Neurosci Lett 518:101-105

McDonagh AF (2010) Controversies in bilirubin biochemistry and their clinical relevance. Semin Fetal Neonat 15:141-147

Morita T, Imai T, Yamaguchi T, Sugiyama T, Katayama S, Yoshino G(2003) Induction of heme oxygenase-1 in monocytes suppresses angiotensin II-elicited chemotactic activity through inhibition of CCR2: role of bilirubin and carbon monoxide generated by the enzyme. Antioxid Redox Signal 5:439-447

Nies A, Keppler D (2007) The apical conjugate efflux pump ABCC2 (MRP2). Pflugers Arch 453:643-659

O'Donnell V, Burkitt MJ(1994) Mitochondrial metabolism of a hydroperoxide to free radicals in human endothelial cells: an electron spin resonance spin-trapping investigation. Biochem J 304:707-713

Ollinger R, Wang H, Yamashita K, Wegiel B, Thomas M, Margreiter R, Bach FH (2007) Therapeutic applications of bilirubin and biliverdin in transplantation. Antioxid 
Redox Signal 9:2175-2185

${ }^{a}$ Pérez LM, Milkiewicz P, Ahmed-Choudhury J, Elias E, Ochoa JE, Sánchez Pozzi EJ, Coleman R, Roma MG(2006) Oxidative stress induces actin-cytoskeletal and tightjunctional alterations in hepatocytes by a $\mathrm{Ca}^{2+}$-dependent, PKC-mediated mechanism: protective effect of PKA. Free Radic Biol Med 40:2005-2017

${ }^{b}$ Pérez LM, Milkiewicz P, Elias E, Coleman R, Sánchez Pozzi EJ, Roma MG(2006) Oxidative stress induces internalization of the bile salt export pump, Bsep, and bile-saltsecretory failure in isolated rat hepatocyte couplets: A role for protein kinase $\mathrm{C}$ and prevention by protein kinase A. Toxicol Sci 91:150-158

Petrov AI, Vatev NT, Atanasova MV(2012) Cholestatic syndrome in viral hepatitis A. Folia Med (Plovdiv) 54:30-35

Roelofsen H, Soroka CJ, Keppler D, Boyer JL(1998) Cyclic AMP stimulates sorting of the canalicular organic anion transporter (Mrp2/cMoat) to the apical domain in hepatocyte couplets. J Cell Sci 111:1137-1145

Rolo AP, Teodoro JS, Palmeira CM(2012) Role of oxidative stress in the pathogenesis of nonalcoholic steatohepatitis. Free Radic Biol Med 52:59-69

Roma MG, Milkiewicz P, Elias E, Coleman R(2000) Control by signaling modulators of the sorting of canalicular transporters in rat hepatocyte couplets: role of the 
cytoskeleton. Hepatology 32:1342-1356

Roma MG, Sanchez Pozzi EJ(2008) Oxidative stress: a radical way to stop making bile. Ann Hepatol 7:16-33

Rost D, Kartenbeck J and Keppler D(1999) Changes in the localization of the rat canalicular conjugate export pump Mrp2 in phalloidin-induced cholestasis. Hepatology 29:814-821

Ryter SW, Morse D, Choi $\operatorname{AM(2007)~Carbon~monoxide~and~bilirubin:~potential~}$ therapies for pulmonary/vascular injury and disease.Am J Respir Cell Mol Biol 36:175182

Sato H, Takeo T, Liu Q, Nakano K, Osanai T, Suga S, Wakui M, Wu J(2009) Hydrogen peroxide mobilizes $\mathrm{Ca}^{2+}$ through two distinct mechanisms in rat hepatocytes. Acta Pharmacol Sin 30:78-89

Schacter BA, Joseph E, Firneisz G(1983) Effect of cholestasis produced by bile duct ligation on hepatic heme and hemoprotein metabolism in rats. Gastroenterology 84:227235

Schmitt M, Kubitz R, Wettstein M, vom Dahl S, Häussinger D(2000) Retrieval of the mrp2 gene encoded conjugate export pump from the canalicular membrane contributes to cholestasis induced by tert-butyl hydroperoxide and chloro-dinitrobenzene. Biol 
Chem 381:487-495

Sedlak TW, Snyder SH(2004) Bilirubin benefits: cellular protection by a biliverdin reductase antioxidant cycle. Pediatrics 113:1776-1782

Sedlak TW, Saleh M, Higginson DS, Paul BD, Juluri KR, Snyder SH(2009) Bilirubin and glutathione have complementary antioxidant and cytoprotective roles. Proc Nat Acad Sci 106:5171-5176

Sorrentino P, Tarantino G, Perrella A, Micheli P, Perrella O, Conca P (2005) A clinicalmorphological study on cholestatic presentation of nonalcoholic fatty liver disease. Dig Dis Sci 50:1130-1135

Stehbens WE(2004) Oxidative stress in viral hepatitis and AIDS.Exp Mol Pathol $77: 121-132$

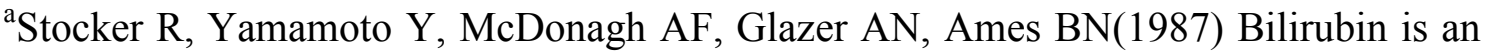
antioxidant of possible physiological importance. Science 235:1043-1046

${ }^{\mathrm{b}}$ Stocker R, Glazer AN, Ames BN(1987) Antioxidant activity of albumin-bound bilirubin. Proc Natl Acad Sci USA 84:5918-5922

Stocker R(2004) Antioxidant activities of bile pigments. Antioxid Redox Signal 6:841849 
Stone V, Johnson G, Wilton J, Coleman R, Chipman J (1994) Effect of oxidative stress and disruption of $\mathrm{Ca}^{2+}$ homeostasis on hepatocytes canalicular function in vitro. Biochem Pharmacol 47:625-632

Talalay P(1960) Enzymatic analysis of steroid hormones. Methods Biochem Anal $8: 119-143$

Tietze F(1969) Enzymic method for quantitative determination of nanogram amounts of total and oxidized glutathione: applications to mammalian blood and other tissues. Anal Biochem 27:502-522

Vitek L (2012) The role of bilirubin in diabetes, metabolic syndrome, and cardiovascular diseases. Front Pharmacol.doi: 10.3389/fphar.2012.00055

Wilton JC, Williams DE, Strain AJ, Parslow RA, Chipman JK, Coleman R (1991) Purification of hepatocyte couplets by centrifugal elutriation. Hepatology 14:180-183

Witting PK, Westerlund C, Stocker R (1996) A rapid and simple screening test for potential inhibitors of tocopherol-mediated peroxidation of LDL lipids. J Lipid Res $37: 853-867$

Yang B and Hill CE(2001) Nifedipine modulation of biliary GSH and GSSG/conjugate efflux in normal and regenerating rat liver. Am J Physiol 281:G85-G94 
Basiglio et al

Zelenka J, Muchova L, Zelenkova M, Vanova K, Vreman HJ, Wong RJ, Vitek L(2012) Intracellular accumulation of bilirubin as a defense mechanism against increased oxidative stress. Biochimie 94:1821-1827 


\section{FIGURE LEGENDS}

Fig 1Protective effect of unconjugated bilirubin (UB) on the oxidative damage induced by tert-butylhydroperoxide(tBuOOH) in isolated rat hepatocytesa) Lipid peroxidation levels.Hepatocytes were incubated in the presence or absence of UB (1.7 $85 \mu \mathrm{M})$, for $15 \mathrm{~min}$. Then, $t \mathrm{BuOOH}(100 \mu \mathrm{M})$ was added to the incubation medium, and cells were incubated for another 15-min period. Lipid peroxidation was evaluated by the generation of malondialdehyde (MDA), as assessed by HPLC. We found that concentrations of UB up to $42.5 \mu \mathrm{M}$ prevented MDA formation. Data are expressed as

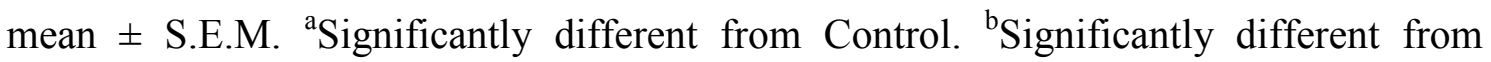
$t \mathrm{BuOOH}(p<0.05 ; n=4)$. b) Intracellular ROS production, assessed by measuring the oxidant-sensitive intracellular production of $2^{\prime}, 7^{\prime}$-dichlorofluorescein from its nonfluorescent, freely diffusible precursor 2',7'- dichlorofluorescin diacetate, by fluorimetric detection. Hepatocytes preloaded with the probe were incubated with or without $\mathrm{UB}(17.1 \mu \mathrm{M}, 15 \mathrm{~min})$, and then with $t \mathrm{BuOOH}(100 \mu \mathrm{M}, 15 \mathrm{~min})$. Data are

expressed as mean \pm S.E.M. ${ }^{\text {a }}$ Significantly different from Control. ${ }^{\mathrm{b}}$ Significantly different from $t \mathrm{BuOOH}(p<0.05 ; n=4)$

Fig 2 Protective effect of unconjugated bilirubin (UB) on the altered function and localization of canalicular transporters induced by tert-butylhydroperoxide(tBuOOH) in isolated rat hepatocytes couplets (IRHC) Couplets were incubated in the presence or absence of UB $(17.1 \mu \mathrm{M}, 15 \mathrm{~min})$, and then in the presence of $t \mathrm{BuOOH}(100 \mu \mathrm{M}, 15$ min). After this treatment, we tested: $\boldsymbol{a})$ Percentage of IRHC (referred to control values) able to accumulate the fluorescent Bsep and Mrp2 substrates, cholyl-glycylamidofluorescein (CGamF) and glutathione-methylfluorescein (GS-MF), respectively, into 
their canalicular vacuoles. The results are expressed as percentages of the control group,

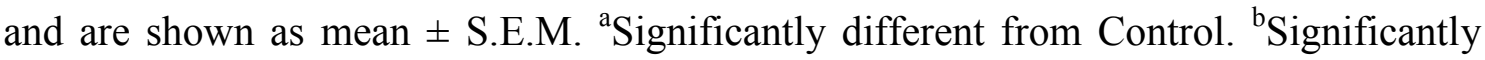
different from $t \mathrm{BuOOH}(p<0.05 ; n=5)$. b) Changes in localization of Bsep (left panel) and Mrp2 (right panel), as illustrated by representative confocal immunofluorescence images. Changes in transporter localization were quantified by densitometric analysis of the fluorescence intensity along a line $(8 \mu \mathrm{m})$, perpendicular to the center of the canalicular vacuole (from +4 to $-4 \mu \mathrm{m}$ ). Representative confocal immunofluorescence images of the localization of Bsep and Mrp2 are also shown. Note that, in control IRHC, both Bsep- and Mrp2-associated fluorescence was localized mainly within the canalicular vacuole, whereas in the group treated with $t \mathrm{BuOOH}$ alone, there was extensive relocalization of the fluorescence from the canalicular zone to the cellular body, thus indicating endocytosis of these canalicular carriers. Statistical analysis of the fluorescence profiles showed a significant change in the IRHC treated with $t \mathrm{BuOOH}$ $(p<0.05$; number of analyzed canalicular vacuoles $>10)$, and this phenomenon was prevented by UB pretreatment for both Bsep and Mrp2

Fig 3Effect of unconjugated bilirubin (UB) on the disruption of actin cytoskeleton induced bytert-butylhydroperoxide (tBuOOH) in isolated rat hepatocyte couplets (IRHC) Couplets were isolated and incubated in the presence or absence of UB (17.1 $\mu \mathrm{M}, 15 \mathrm{~min})$, and then exposed tot $\mathrm{BuOOH}(100 \mu \mathrm{M}, 15 \mathrm{~min})$. At the end of the experiments, changes in actin localization were evaluated by (a) bleb formation, as assessed by phase-contrast microscopy, and (b) actin staining with fluorescently-labeled phalloidin; canalicular localization of actin was then quantified by assessing the proportion of fluorescence intensity localized in the pericanalicular zone. Data are 


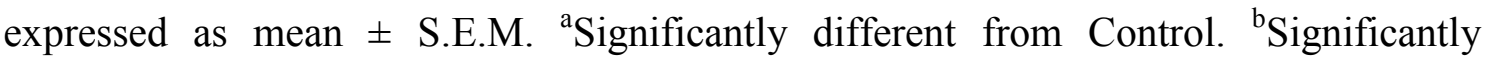
different from $t \mathrm{BuOOH}(p<0.05 ; n=4)$

Fig 4Effect of unconjugated bilirubin (UB) on the alterations in biliary secretory function induced by tert-butylhydroperoxide (tBuOOH) in the isolated perfused rat liver (IPRL) After a 20-min equilibration period, UB (or its vehicle, 3\% BSA, in Control and $t \mathrm{BuOOH}$ alone groups) was added to the reservoir so as to reach a $2.1 \mu \mathrm{M}$ final concentration. Fifteen min after UB addition, a 10-min bile sample was collected, followed by a shift of the perfusion medium to another one containing $75 \mu \mathrm{M} t \mathrm{BuOOH}$ (or DMSO in Control and UB alone groups) or $75 \mu \mathrm{M} t \mathrm{BuOOH}+2.1 \mu \mathrm{M}$ UB. After a 15-min perfusion with $t \mathrm{BuOOH}$ or $t \mathrm{BuOOH}+\mathrm{UB}$, the perfusion medium was shifted back to the basal medium, containing neither UB nor $t \mathrm{BuOOH}$. Bile samples were collected at 10-min intervals for $60 \mathrm{~min}$. Temporal changes of the following parameters (expressed as percentages of their mean basal values recorded at the onset of the perfusion process) were assessed, namely: a) the ratio of oxidized glutathione (as glutathione disulfide, GSSG) tototal (reduced + oxidized) glutathione (GSHt); b) bile flow; $c$ ) biliary excretion of bile salts, resulting from having added a traceable dose of the Bsep substrate taurocholate to the perfusion medium; $\boldsymbol{d}$ ) biliary excretion of the Mrp2 substrate dinitrophenyl-glutathione (GS-DNP) resulting from having added chloro-dinitrobenzene (CDNB) to the perfusion medium. Note that UB markedly prevented the abrupt increase in the GSSG/GSHt relation, the drop in bile flow, and the decrease in the excretion of both bile salts and GS-DNP induced by $t \mathrm{BuOOH}$. Data are expressed as mean \pm S.E.M. *Significantly different from Control, UB and $\mathrm{UB}+t \mathrm{BuOOH}(p<0.05, n=5)$ 
Fig 5Endocytic internalization of Bsep and Mrp2 and disruption of F-actin cytoskeleton induced by tert-butylhydroperoxide (tBuOOH), and their prevention by unconjugated bilirubin (UB) in isolated perfused rat liver $($ IPRL)Representativeconfocal images illustrating the endocytic internalization of Bsep and Mrp2 induced by $t \mathrm{BuOOH}$, and its prevention by UB. The images were taken from IPRL samples, excised $20 \mathrm{~min}$ after the onset of $t \mathrm{BuOOH}$ perfusion. $\boldsymbol{a}$ )Co-staining of Bsep (green) and F-actin (red); b)Co-staining of Mrp2 (green) and F-actin (red). In both sets of micrographs, the following groups are shown: $\boldsymbol{A}$ ) Control (vehicles only), $\boldsymbol{B}$ ) UB $(2.1 \mu \mathrm{M}, 15 \mathrm{~min}), \boldsymbol{C}) t \mathrm{BuOOH}(75 \mu \mathrm{M}, 10 \mathrm{~min}), \boldsymbol{D}) \mathrm{UB}(2.1 \mu \mathrm{M}, 15 \mathrm{~min})+t \mathrm{BuOOH}(75$ $\mu \mathrm{M}, 10 \mathrm{~min})$. Note that, in control livers, both Bsep and Mrp2 were mainly confined to the canalicular space, while in livers treated with $t \mathrm{BuOOH}$, relocalization of both Bsep and Mrp2 from the canalicular space to the pericanalicular area became apparent, thus suggesting endocytic internalization of the transporters. UB prevented this phenomenon, as illustrated by a control-like pattern of Bsep and Mrp2 distribution in livers pre-treated with UB. Note also that pericanalicular localization of F-actin was only apparent in control livers, while a severe disarrangement of F-actin was observed in livers perfused with $t \mathrm{BuOOH}$. UB partially prevented the oxidative stress-induced disruption on F-actin localization, thus explaining the protective effects of UBon canalicular transporters localization and function

Fig 6Effect of unconjugated bilirubin (UB) on protein kinase C (PKC) activation by tert-butylhydroperoxide (tВиООН) The upper panels are representative western blots of membrane PKC and cytosolic PKC obtained from whole cellular lysates of isolated 
Basiglio et al

rat hepatocytes incubated with $t \mathrm{BuOOH}(100 \mathrm{lM})$ for $15 \mathrm{~min}$ or with $t \mathrm{BuOOH}(100 \mathrm{lM})$ for $15 \mathrm{~min}$ in cells pretreated with UB for $15 \mathrm{~min}$. The lower panel shows the PKC activation status (expressed as the membrane-localized PKC to total PKC ratio) for each experimental condition. An arbitrary value of 100 was assigned to the band of highest densitometric intensity in every western blot before the membrane-localized PKC to total PKC ratio was calculated. Data are expressed as mean \pm S.E.M. ${ }^{\text {a Significantly }}$ different from Control. ${ }^{\mathrm{b}}$ Significantly different from $t \mathrm{BuOOH}(p<0.05 ; n=4)$ 